\title{
APPLICATION-ORIENTED DESCRIPTION OF THE QUALITY OF ELECTRONIC COMPONENTS (ppm CONCEPT) $\dagger$
}

\author{
WOLFGANG JUNGHANS \\ Valvo Unternehmensbereich Bauelemente der Philips GmbH, Hamburg
}

(Received December 20, 1980; in final form January 2, 1981)

\begin{abstract}
Traditionally the production quality of components is described by "acceptable quality levels" (AQL). Agreements, referring to this principle, determine the acceptance or refusal of batches that have been sample tested. An acceptable quality of level is interpreted as a tolerated limit. In reality true values of quality must be better. An AQL value cannot define a real quality level. Furthermore, increasing requirements for component quality lead to low figures for the permissible amount of faulty devices in batches, and the method of sample testing becomes insufficient.

For these reasons it seems to be of major importance to establish a better standard for the definition of quality. The application oriented description of quality data offers a possible solution. A good exchange of experience between supplier and user, based on a close and quality loop, is necessary.

For contractual purposes AQL values remain important, but their meaning needs a new interpretation.
\end{abstract}

\section{INTRODUCTION}

Industrial supplies as well as all services are, in general based on contracts, and they may be agreed upon in general or for an individual case. In the majority of these contracts for future delivery, aspects of quality are covered by rules of warranty. Thus in any consideration of agreements for purchase of electronic components a combination of technical and contractual aspects is involved.

Unfortunately an unrestricted safe $100 \%$ tested quality product cannot be manufactured. On the other hand the customer has the right to be supplied with faultless products. Thus technically oriented agreements are needed which traditionally include the definition of AQL-values required for the 'zero hour' production quality, the 'conformity'. This is possible, provided that the receiver of products applies sampling methods in the goods inwards inspection.

\section{AQL AGREEMENTS}

As long as the term quality in both context and concept did not have today's major importance, AQL-agreements fulfilled both contractual and technical requirements respectively.

However, an AQL-agreement as such cannot satisfy

\footnotetext{
$\nmid$ Paper originally given in the Technical Papers programme of Electronica, Munich, November, 1980.
}

today's increased requirements for quality. In particular for complex devices for which a high number of test characteristics can be identified, the inspection specification must be agreed upon. In this respect treatments are often unprecise. For example, it can be understood that an Original Equipment Manufacturer (OEM) regards components as defective that do not function in his equipment, but there remains the question as to whether he has identified a component's real fault or not. This example will be referred to later.

$A Q L$ values are figures which determine under which defined conditions a lot which has been sampletested will be accepted or not. The test-result of the individual lot does not say, however, with sufficient accuracy how good or bad it is.

The connection with the quality, or more precisely with the number of faulty devices in the lot, is defined by mathematical statistics, provided a sufficiently large number of lots are tested. ${ }^{1,2,3,4}$

In principle the acceptance probability of passing a production batch is fixed at around $95 \%$ if full advantage is made of the agreed AQL value. This means that there is a $5 \%$ chance that the manufacturer will re-inspect a batch of sufficient quality because of the results of the tests on a sample group from the batch. It also means that the customer for the components has a chance of receiving batches which have been passed from the sampling results but that have finite \% defects in the batches. (Thus for a particular operating characteristic there will be a $93 \%$ 
chance that $10 \%$ are defective, and a $16 \%$ chance that $40 \%$ are defective, etc.) This latter situation is not acceptable either from the logistical or economical point of view. From this it follows that the real AQL of the manufactured components must be much better than is apparent from the published agreed value.

The problem cannot be solved by more severe AQL-values. Below AQL $=0.1$ a sampling procedure cannot be performed economically as this would lead to $100 \%$ inspections.

Disregarding the statement that AQL-values do not define an actual supply quality, there are further important arguments for applying sampling tests in the goods inwards inspection in order to make a decision as to whether the supplied lots are to be accepted or refused. These are:-

i) to prevent further use in the case of incorrect supplies.

ii) to assure that no lots with an excessively high number of faulty devices are used.

iii) to assure that any eventual claim for poor quality goods be lodged within an agreed time limit.

iv) to perform part of the measurements required in connection with product liability.

\section{APPLICATION ORIENTED TESTING}

Requirements for the number of faulty devices in the received batch in the order of a few faults per million components tested (ppm), are becoming common. How can such requirements be handled if sampling schemes are unsuccessful?

Initially it has been mentioned that all components supplied must work in the equipment without any restriction and that this cannot be attained definitely by formal conformity tests of characteristics. In other words, the quality of components must refer to the application. An answer to this requirement leads to the so-called ppm-concept.

Figure 1 shows the passage of components ready for use from their $100 \%$ final inspection to the first switching on and test-run of the completed equipment. After the final inspection an attributive sampling procedure of the goods ready for dispatch is performed in order to check the effectiveness of the preceding tests. Results of conformity data which are found here are accumulated. In order to achieve reliable figures for the process average calculated in this way a sufficiently high number of units must be tested. Only structurally similar components can be grouped together, i.e. those with conforming quality features due to mutual construction technology.

Ppm considerations result in the necessity of operating with large numbers of components. For example, for a confidence level of $90 \%$ in a quality target of $100 \mathrm{ppm}, 10^{5}$ components must be tested to achieve a confidence interval of between 70 and 154 ppm. Such figures:

Component Manufacturer

Equipment manufacturer

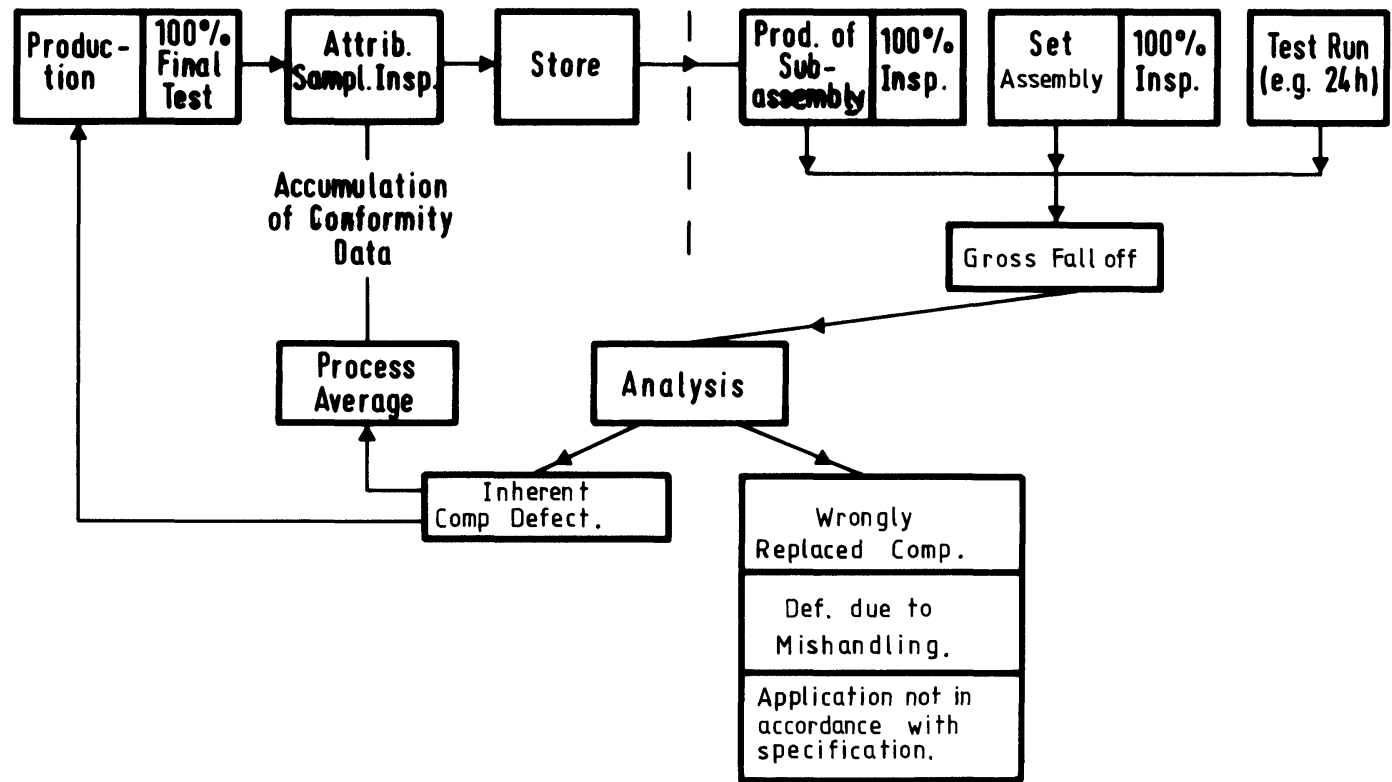

FIGURE 1 Diagram demonstrating the closed nature of the quality loop . 
- describe the true process average.

- result from a defined period of test (i.e. 3 months).

- are an average value.

- refer to past performance only.

- will not be found in the future to individual supply lots.

- are not applicable to contractual agreements in the future.

However they are realistic values to be expected for future supplies.

From experience these figures cannot always be obtained by the OEM. This applies in particular to complex components. Integrated circuits belong to these complex components for which the result of an analysis from the year 1979 is shown in Figure 2. Areas in which the component supplier, the equipment manufacturer or both together are involved, can be seen. Furthermore it can be noticed that for rejection proportions of around $40 \%$, the amount of difficulties due to not organizing the test programmes with reference to the application requirements is much

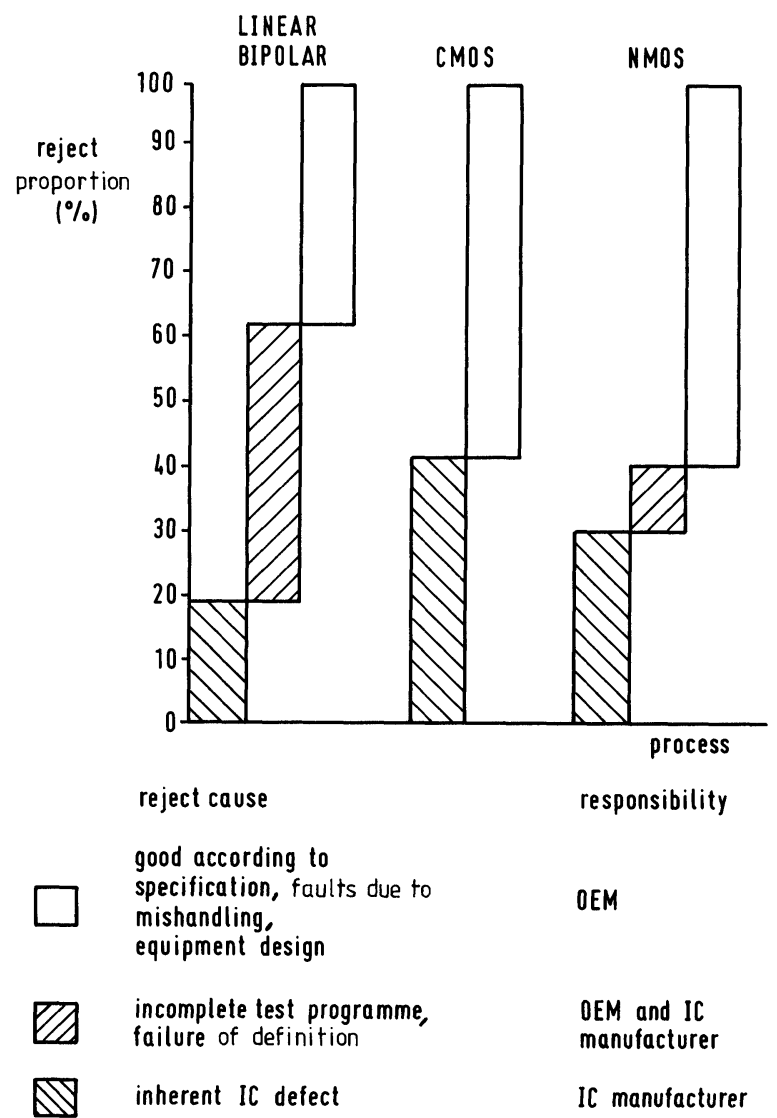

FIGURE 2 Distribution of causes of IC-rejections during 1979. higher for linear bipolar circuits than in the digital area of CMOS and NMOS device circuits. The reason for this can be found in the better defined circuit application design rules for this latter group of components. In particular, for integrated circuits in the area of analogue applications it is more difficult to define specifications in order to cover all possible applications as well as all the exceptional working conditions of the apparatus.

There will always remain an undefined field. This applies to the specifications and test programmes of the component manufacturer as well as for those written by the users. Such kinds of problems can only be recognized and revised if manufacturer and user co-operate technically. This must be based on an accurate analysis of the production rejects in the equipment manufacturer's production, the so-called fell-off.

In Figure 2 it can also be seen that rejects are shown which refer to either the component manufacturer or equipment manufacturer respectively. Such a definite association is doubtlessly correct for clearly identified failures which can be of human or machine nature. Collaboration between supplier and user is therefore essential in this area. Within this group of faults, however, equipment can fail due to the cumulative effect of faults in different devices rather than due to one device failure.

Not every fault must inevitably cause a failure. A weak point due to a component may result in an equipment failure only in connection with certain possibly complex usages. In this case the components manufacturer will not be at fault. On the other hand not all infringements of circuit design rules will lead to a failure of components, and the equipment manufacturer will not be liable either.

The proportion of components rejected during the manufacturing process of equipment as well as later on during practical use leads to a 'worst case' consideration that can be regarded as a consequence of a chain of accidents, in other words not as a strong determining causality-chain.

If we are dealing here at least partly with a stochastic process, it is clear that failure mechanisms in the ppm-area reasoned in this way can only be understood by a sufficiently close co-operation between component and equipment-manufacturers.

It will not be possible for each manufacturer to correspond with each user of his products at a high technical level. However it is important that a sufficient number of companies with adequate qualifications, do co-operate. The result will be advantageous to the entire industry. 


\section{CONCLUSION}

It has been shown that realistic values of $\mathrm{AQL}$ are not practicable, so that quality at the ppm level can only be achieved by the co-operation of the component and equipment industries in terms of a technical partnership. Such a procedure is of more practical use than the formal demarcating and contractual handling of the problem.

\section{REFERENCES}

1. Publications of the Deutsche Gesellschaft fur Qualität, Beuth Verlag, GmbH. (Berlin (D); Köln (D)).

2. BS6000: 1972. Guide to the use of sampling procedures and tables for inspection by attributes. British Standards Institution.

3. BS6001: 1972. Specification for sampling procedures and tables for inspection by attributes. British Standards Institution.

4. BS6002: 1979. Specification for sampling procedures and charts for inspection by variables for percent defective. British Standards Institution. 

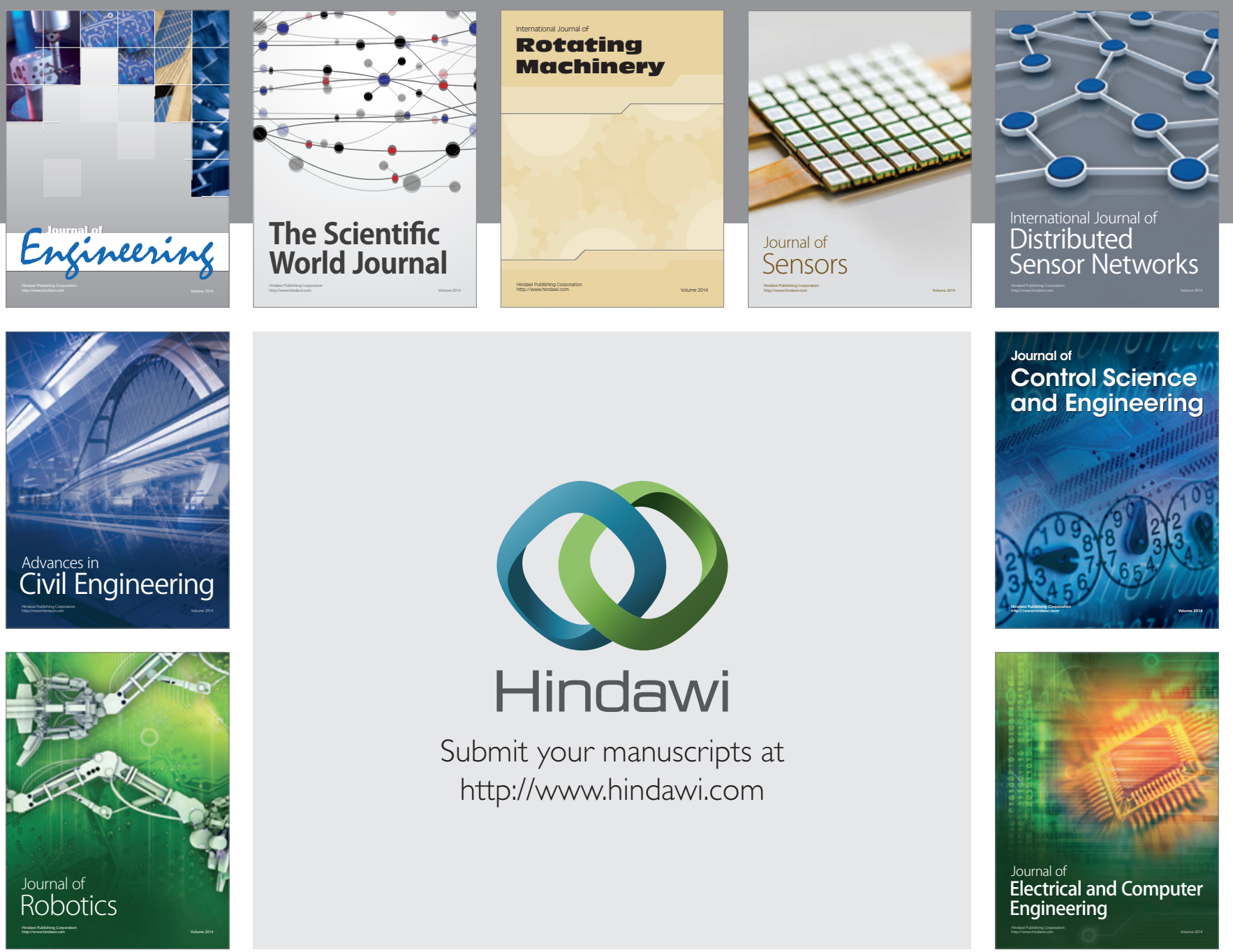

Submit your manuscripts at

http://www.hindawi.com
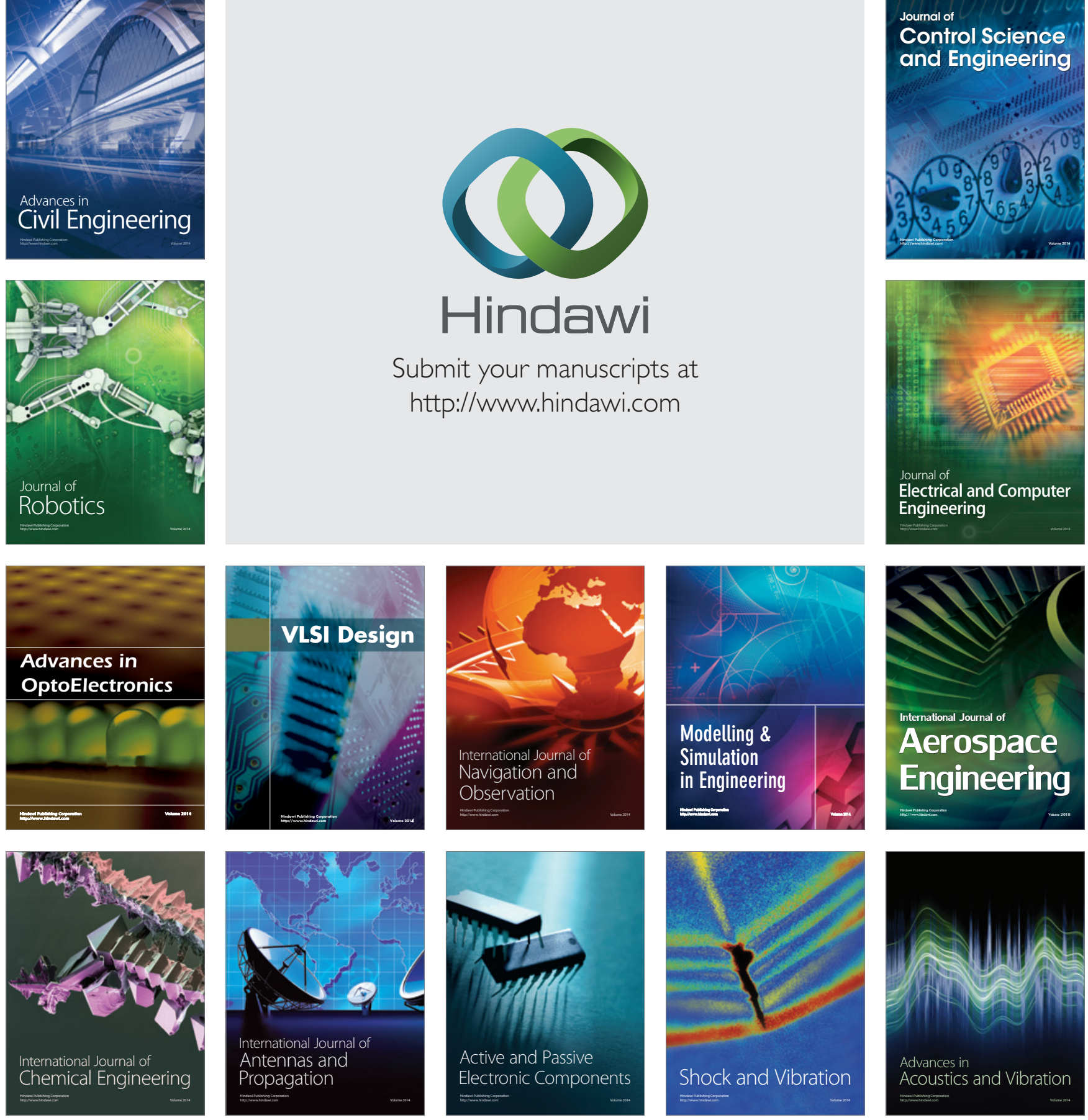